PROCEEDINGS OF THE

AMERICAN MATHEMATICAL SOCIETY

Volume 133, Number 5, Pages 1257-1265

S 0002-9939(04)07863-3

Article electronically published on December 15, 2004

\title{
EULER'S INTEGRALS AND MULTIPLE SINE FUNCTIONS
}

\author{
SHIN-YA KOYAMA AND NOBUSHIGE KUROKAWA
}

(Communicated by David E. Rohrlich)

\begin{abstract}
We show that Euler's famous integrals whose integrands contain the logarithm of the sine function are expressed via multiple sine functions.
\end{abstract}

\section{INTRODUCTION}

Euler studied the definite integrals $\int_{0}^{\frac{\pi}{2}} x^{n} \log (\sin x) d x$ for $n=0$ and 1. In [E1] (1769), he proved the famous result

$$
\int_{0}^{\frac{\pi}{2}} \log (\sin x) d x=-\frac{\pi}{2} \log 2
$$

which is frequently explained as an example of tricky integrals in analysis courses. A bit later, Euler [E2] (1772) stated that

$$
\begin{aligned}
\int_{0}^{\frac{\pi}{2}} x \log (\sin x) d x & =\frac{1}{2}\left(\sum_{\substack{n=1 \\
n \text { :odd }}}^{\infty} \frac{1}{n^{3}}-\frac{\pi^{2}}{4} \log 2\right) \\
& =\frac{7}{16} \zeta(3)-\frac{\pi^{2}}{8} \log 2 .
\end{aligned}
$$

Euler proved (1) by using

$$
\log (\sin x)=-\sum_{n=1}^{\infty} \frac{\cos (2 n x)}{n}-\log 2
$$

for $0<x<\pi$. The actual integration is obvious since

$$
\int_{0}^{\frac{\pi}{2}} \cos (2 n x) d x=0
$$

for $n=1,2,3, \cdots$. Hence, the original proof of Euler is not tricky contrary to the usual explanation. In the case of (2), Euler primarily wanted to calculate the value $\zeta(3)$. He started from the divergent series expression

$$
\begin{aligned}
\zeta(3) & =-4 \pi^{2} \zeta^{\prime}(-2) \\
& =4 \pi^{2} \sum_{n=1}^{\infty} n^{2} \log n
\end{aligned}
$$

Received by the editors August 4, 2003.

2000 Mathematics Subject Classification. Primary 11M06.

(C)2004 American Mathematical Society Reverts to public domain 28 years from publication 
and by investigating it he reached (2). Thus his arguments are difficult to follow and literally invalid. Moreover Euler did not prove the functional equation $\zeta(3)=$ $-4 \pi^{2} \zeta^{\prime}(-2)$ conjectured by himself in [E3]. We notice that when we use (3) we can give a secure calculation for (2):

$$
\int_{0}^{\frac{\pi}{2}} x \log (\sin x) d x=-\sum_{n=1}^{\infty} \frac{1}{n} \int_{0}^{\frac{\pi}{2}} x \cos (2 n x) d x-\frac{\pi^{2}}{8} \log 2
$$

with integration by parts

$$
\int_{0}^{\frac{\pi}{2}} x \cos (2 n x) d x=\left\{\begin{array}{cl}
-\frac{1}{2 n^{2}} & \text { if } n \text { is odd } \\
0 & \text { if } n \text { is even. }
\end{array}\right.
$$

It might be remarkable that Euler missed this way.

In this paper we investigate definite integrals

$$
\int_{0}^{x} \theta^{r-2} \log (\sin \theta) d \theta
$$

for $r=2,3,4, \cdots$ containing Euler's case $x=\pi / 2$ from the point of view of multiple sine functions. Let

$$
\begin{aligned}
\mathcal{S}_{r}(x) & =e^{\frac{x^{r}-1}{r-1}} \prod_{\substack{n=-\infty \\
n \neq 0}}^{\infty} P_{r}\left(\frac{x}{n}\right)^{n^{r-1}} \\
& =e^{\frac{x^{r-1}}{r-1}} \prod_{n=1}^{\infty}\left(P_{r}\left(\frac{x}{n}\right) P_{r}\left(-\frac{x}{n}\right)^{(-1)^{r-1}}\right)^{n^{r-1}}
\end{aligned}
$$

be the multiple sine function studied in [K1, K2, KK1, KK2, KOW, KW], where

$$
P_{r}(u)=(1-u) \exp \left(u+\frac{u^{2}}{2}+\cdots+\frac{u^{r}}{r}\right) .
$$

For example,

$$
\begin{gathered}
\mathcal{S}_{2}(x)=e^{x} \prod_{n=1}^{\infty}\left(\left(\frac{1-\frac{x}{n}}{1+\frac{x}{n}}\right)^{n} e^{2 x}\right), \\
\mathcal{S}_{3}(x)=e^{\frac{x^{2}}{2}} \prod_{n=1}^{\infty}\left(\left(1-\frac{x^{2}}{n^{2}}\right)^{n^{2}} e^{x^{2}}\right)
\end{gathered}
$$

and

$$
\mathcal{S}_{4}(x)=e^{\frac{x^{3}}{3}} \prod_{n=1}^{\infty}\left(\left(\frac{1-\frac{x}{n}}{1+\frac{x}{n}}\right)^{n^{3}} e^{2 n^{2} x+\frac{2}{3} x^{3}}\right) .
$$

Then we show the following result.

Theorem 1. For $0 \leq x<\pi$ and for $r=2,3,4, \ldots$, we have

$$
\int_{0}^{x} \theta^{r-2} \log (\sin \theta) d \theta=\frac{x^{r-1}}{r-1} \log (\sin x)-\frac{\pi^{r-1}}{r-1} \log \mathcal{S}_{r}\left(\frac{x}{\pi}\right) .
$$


In particular we have:

Theorem 2. For $r=2,3,4, \ldots$,

$$
\int_{0}^{\frac{\pi}{2}} \theta^{r-2} \log (\sin \theta) d \theta=-\frac{\pi^{r-1}}{r-1} \log \mathcal{S}_{r}\left(\frac{1}{2}\right) .
$$

Examples.

$$
\begin{aligned}
\int_{0}^{\frac{\pi}{2}} \log (\sin \theta) d \theta & =-\pi \log \mathcal{S}_{2}\left(\frac{1}{2}\right) \\
& =-\pi \log \left(e^{\frac{1}{2}} \prod_{n=1}^{\infty}\left(\left(\frac{2 n-1}{2 n+1}\right)^{n} e\right)\right) \\
\int_{0}^{\frac{\pi}{2}} \theta \log (\sin \theta) d \theta & =-\frac{\pi^{2}}{2} \log \mathcal{S}_{3}\left(\frac{1}{2}\right) \\
& =-\frac{\pi^{2}}{2} \log \left(e^{\frac{1}{8}} \prod_{n=1}^{\infty}\left(\left(1-\frac{1}{4 n^{2}}\right)^{n^{2}} e^{\frac{1}{4}}\right)\right), \\
\int_{0}^{\frac{\pi}{2}} \theta^{2} \log (\sin \theta) d \theta= & -\frac{\pi^{3}}{3} \log \mathcal{S}_{4}\left(\frac{1}{2}\right)^{-} \\
= & -\frac{\pi^{3}}{3} \log \left(e^{\frac{1}{24}} \prod_{n=1}^{\infty}\left(\left(\frac{2 n-1}{2 n+1}\right)^{n^{3}} \exp \left(n^{2}+\frac{1}{12}\right)\right)\right) .
\end{aligned}
$$

We notice that we have $\mathcal{S}_{2}\left(\frac{1}{2}\right)=\sqrt{2}$ and $\mathcal{S}_{3}\left(\frac{1}{2}\right)=2^{\frac{1}{4}} \exp \left(-\frac{7 \zeta(3)}{8 \pi^{2}}\right)$ from Euler's results (11) and (2) combined with (4) and (5). We demonstrate a calculation of the special value $\mathcal{S}_{4}\left(\frac{1}{2}\right)$ from the product expression directly as follows.

\section{Theorem 3.}

$$
\mathcal{S}_{4}\left(\frac{1}{2}\right)=2^{\frac{1}{8}} \exp \left(-\frac{9 \zeta(3)}{16 \pi^{2}}\right)
$$

and

$$
\int_{0}^{\frac{\pi}{2}} \theta^{2} \log (\sin \theta) d \theta=\frac{3 \pi}{16} \zeta(3)-\frac{\pi^{3}}{24} \log 2 .
$$

In our calculation a generalization of the Stirling formula is crucial.

\section{Multiple sine functions}

To make this paper self-contained we prove some basic properties of multiple sine functions. For general background we refer to [KK1, KK2, [KOW, $\mathrm{M}]$.

Proposition 1. For $r=2,3,4, \ldots, \mathcal{S}_{r}(x)$ is a meromorphic function in $x \in \mathbf{C}$ and it satisfies

$$
\frac{\mathcal{S}_{r}^{\prime}(x)}{\mathcal{S}_{r}(x)}=\pi x^{r-1} \cot (\pi x)
$$


Proof. The fact that $\mathcal{S}_{r}(x)$ is a meromorphic function in $x \in \mathbf{C}$ (and its order as a meromorphic function being $r$ ) is seen from the product expression defining $\mathcal{S}_{r}(x)$. Let us calculate the logarithmic derivative. From

$$
\mathcal{S}_{r}(x)=e^{\frac{x^{r-1}}{r-1}} \prod_{n=1}^{\infty}\left(P_{r}\left(\frac{x}{n}\right) P_{r}\left(-\frac{x}{n}\right)^{(-1)^{r-1}}\right)^{n^{r-1}}
$$

we have

$$
\begin{aligned}
\log \mathcal{S}_{r}(x)= & \frac{x^{r-1}}{r-1}+\sum_{n=1}^{\infty} n^{r-1}\left(\log P_{r}\left(\frac{x}{n}\right)+(-1)^{r-1} \log P_{r}\left(-\frac{x}{n}\right)\right) \\
= & \frac{x^{r-1}}{r-1}+\sum_{n=1}^{\infty} n^{r-1}\left(\log \left(1-\frac{x}{n}\right)+(-1)^{r-1} \log \left(1+\frac{x}{n}\right)\right. \\
& +\left(\frac{x}{n}+\frac{1}{2}\left(\frac{x}{n}\right)^{2}+\cdots+\frac{1}{r}\left(\frac{x}{n}\right)^{r}\right) \\
& \left.+(-1)^{r-1}\left(\left(\frac{-x}{n}\right)+\frac{1}{2}\left(\frac{-x}{n}\right)^{2}+\cdots+\frac{1}{r}\left(\frac{-x}{n}\right)^{r}\right)\right) .
\end{aligned}
$$

Hence

$$
\begin{aligned}
\frac{\mathcal{S}_{r}^{\prime}(x)}{\mathcal{S}_{r}(x)}=x^{r-2}+\sum_{n=1}^{\infty} n^{r-1}\left(\frac{1}{x-n}\right. & +\frac{(-1)^{r-1}}{x+n}+\left(\frac{1}{n}+\frac{x}{n^{2}}+\cdots+\frac{x^{r-1}}{n^{r}}\right) \\
& \left.+(-1)^{r-1}\left(-\frac{1}{n}+\frac{x}{n^{2}}+\cdots+(-1)^{r} \frac{x^{r-1}}{n^{r}}\right)\right) .
\end{aligned}
$$

Here

and

$$
\frac{1}{n}+\frac{x}{n^{2}}+\cdots+\frac{x^{r-1}}{n^{r}}=\frac{\left(\frac{x}{n}\right)^{r}-1}{x-n}
$$

$$
-\frac{1}{n}+\frac{x}{n^{2}}+\cdots+(-1)^{r} \frac{x^{r-1}}{n^{r}}=\frac{(-1)^{r}\left(\frac{x}{n}\right)^{r}-1}{x+n} .
$$

Thus

$$
\begin{aligned}
\frac{\mathcal{S}_{r}^{\prime}(x)}{\mathcal{S}_{r}(x)} & =x^{r-2}+\sum_{n=1}^{\infty} n^{r-1}\left(\frac{\left(\frac{x}{n}\right)^{r}}{x-n}-\frac{\left(\frac{x}{n}\right)^{r}}{x+n}\right) \\
& =x^{r-2}+\sum_{n=1}^{\infty} \frac{2 x^{r}}{x^{2}-n^{2}} \\
& =\pi x^{r-1} \cot (\pi x),
\end{aligned}
$$

where we used

$$
\pi \cot (\pi x)=\frac{1}{x}+\sum_{n=1}^{\infty} \frac{2 x}{x^{2}-n^{2}} .
$$

Proposition 2. For $0 \leq x<1$ and for $r=2,3,4, \ldots$,

$$
\log \mathcal{S}_{r}(x)=\int_{0}^{x} \pi t^{r-1} \cot (\pi t) d t .
$$

Proof. Since $\mathcal{S}_{r}(0)=1$, both sides are 0 at $x=0$. Hence it is sufficient to remark that the differentiations of both sides are $\pi x^{r-1} \cot (\pi x)$ from Proposition 1 . 


\section{EULER's INTEGRALS}

Using Proposition 2 we show Theorems 1 and 2

Proof of Theorems 1 and Q . By integration by parts in

$$
\log \mathcal{S}_{r}(x)=\int_{0}^{x} \pi t^{r-1} \cot (\pi t) d t
$$

we have

$$
\begin{aligned}
\log \mathcal{S}_{r}(x) & =\left[t^{r-1} \log (\sin \pi t)\right]_{0}^{x}-\int_{0}^{x}(r-1) t^{r-2} \log (\sin \pi t) d t \\
& =x^{r-1} \log (\sin \pi x)-(r-1) \int_{0}^{x} t^{r-2} \log (\sin \pi t) d t .
\end{aligned}
$$

Hence changing the variable to $\theta=\pi t$ in the integral, we have

$$
\log \mathcal{S}_{r}(x)=x^{r-1} \log (\sin \pi x)-\frac{r-1}{\pi^{r-1}} \int_{0}^{\pi x} \theta^{r-2} \log (\sin \theta) d \theta .
$$

This gives Theorem 1 Then, letting $x=1 / 2$ we have Theorem 2 ,

\section{Examples.}

$$
\begin{gathered}
\int_{0}^{\frac{\pi}{4}} \log (\sin \theta) d \theta=-\frac{\pi}{8} \log 2-\pi \log \mathcal{S}_{2}\left(\frac{1}{4}\right), \\
\int_{0}^{\frac{\pi}{3}} \log (\sin \theta) d \theta=\frac{\pi}{3} \log \frac{\sqrt{3}}{2}-\pi \log \mathcal{S}_{2}\left(\frac{1}{3}\right), \\
\int_{0}^{\frac{\pi}{4}} \theta \log (\sin \theta) d \theta=-\frac{\pi^{2}}{64} \log 2-\frac{\pi^{2}}{2} \log \mathcal{S}_{3}\left(\frac{1}{4}\right), \\
\int_{0}^{\frac{\pi}{3}} \theta \log (\sin \theta) d \theta=\frac{\pi^{2}}{18} \log \frac{\sqrt{3}}{2}-\frac{\pi^{2}}{2} \log \mathcal{S}_{3}\left(\frac{1}{3}\right) .
\end{gathered}
$$

\section{A calculation of the special value}

Proof of Theorem 3. Since

$$
\mathcal{S}_{4}\left(\frac{1}{2}\right)=e^{\frac{1}{24}} \prod_{n=1}^{\infty}\left(\left(\frac{2 n-1}{2 n+1}\right)^{n^{3}} \exp \left(n^{2}+\frac{1}{12}\right)\right),
$$

we put

$$
\begin{aligned}
A_{N}= & e^{\frac{1}{24}} \prod_{n=1}^{N}\left(\left(\frac{2 n-1}{2 n+1}\right)^{n^{3}} \exp \left(n^{2}+\frac{1}{12}\right)\right) \\
= & \exp \left(\frac{1}{24}+\left(1^{2}+\cdots+N^{2}\right)+\frac{N}{12}\right) \times \prod_{n=1}^{N}(2 n-1)^{n^{3}-(n-1)^{3}} \\
& \times(2 N+1)^{-N^{3}}
\end{aligned}
$$

and show

$$
\lim _{N \rightarrow \infty} A_{N}=2^{\frac{1}{8}} \exp \left(\frac{9}{4} \zeta^{\prime}(-2)\right)=2^{\frac{1}{8}} \exp \left(-\frac{9 \zeta(3)}{16 \pi^{2}}\right) .
$$


Then the value of the integral follows from ([6). We use the Stirling formula

$$
N !=\prod_{n=1}^{N} n \sim \sqrt{2 \pi} N^{N+\frac{1}{2}} e^{-N}
$$

and its generalization

$$
\prod_{n=1}^{N} n^{n^{2}} \sim \exp \left(-\zeta^{\prime}(-2)\right) N^{\frac{N^{3}}{3}+\frac{N^{2}}{2}+\frac{N}{6}} e^{-\frac{N^{3}}{9}+\frac{N}{12}},
$$

which follow from the Euler-Maclaurin summation formula for $\zeta^{\prime}(s)$ :

$$
\begin{aligned}
\zeta^{\prime}(s)=\lim _{N \rightarrow \infty}\left(-\sum_{n=1}^{N} n^{-s} \log n\right. & +\frac{N^{1-s} \log N}{1-s}-\frac{N^{1-s}}{(1-s)^{2}} \\
& \left.+\frac{1}{2} N^{-s} \log N-\frac{s}{12} N^{-s-1} \log N+\frac{1}{12} N^{-s-1}\right)
\end{aligned}
$$

valid in $\operatorname{Re}(s)>-3$. We refer to Hardy $[\mathrm{H}]$, Chap. XIII, for the Euler-Maclaurin summation formula and its applications. Then, letting $s=0$ and -2 we see

$$
\begin{aligned}
\log \sqrt{2 \pi}=-\zeta^{\prime}(0) & =\lim _{N \rightarrow \infty}\left(\sum_{n=1}^{N} \log n-\left(\left(N+\frac{1}{2}\right) \log N-N\right)\right) \\
& =\lim _{N \rightarrow \infty} \log \left(\frac{N !}{N^{N+\frac{1}{2}} e^{-N}}\right)
\end{aligned}
$$

and

$$
\begin{aligned}
-\zeta^{\prime}(-2) & =\lim _{N \rightarrow \infty}\left(\sum_{n=1}^{N} n^{2} \log n-\left(\left(\frac{N^{3}}{3}+\frac{N^{2}}{2}+\frac{N}{6}\right) \log N-\frac{N^{3}}{9}+\frac{N}{12}\right)\right) \\
& =\lim _{N \rightarrow \infty} \log \left(\frac{\prod_{n=1}^{N} n^{n^{2}}}{N^{\frac{N^{3}}{3}+\frac{N^{2}}{2}+\frac{N}{6}} e^{-\frac{N^{3}}{9}+\frac{N}{12}}}\right) .
\end{aligned}
$$

Using

$$
\begin{aligned}
\prod_{n=1}^{N}(2 n-1)^{n^{3}-(n-1)^{3}} & =\prod_{n=1}^{N}(2 n-1)^{3 n^{2}-3 n+1} \\
& =\left(\prod_{n=1}^{N}(2 n-1)^{(2 n-1)^{2}}\right)^{\frac{3}{4}} \times\left(\prod_{n=1}^{N}(2 n-1)\right)^{\frac{1}{4}} \\
& =\left(\frac{\prod_{n=1}^{2 N} n^{n^{2}}}{\prod_{n=1}^{N}(2 n)^{(2 n)^{2}}}\right)^{\frac{3}{4}} \times\left(\frac{\prod_{n=1}^{2 N} n}{\prod_{n=1}^{N}(2 n)}\right)^{\frac{1}{4}}
\end{aligned}
$$


and the (generalized) Stirling formulas

$$
\begin{gathered}
\prod_{n=1}^{2 N} n^{n^{2}} \sim e^{-\zeta^{\prime}(-2)}(2 N)^{\frac{8 N^{3}}{3}+2 N^{2}+\frac{N}{3}} e^{-\frac{8 N^{3}}{9}+\frac{N}{6}}, \\
\prod_{n=1}^{N}(2 n)^{(2 n)^{2}}=2^{4\left(1^{2}+\cdots+N^{2}\right)}\left(\prod_{n=1}^{N} n^{n^{2}}\right)^{4} \\
\sim 2^{\frac{2}{3} N(N+1)(2 N+1)}\left(e^{-\zeta^{\prime}(-2)} N^{\frac{N^{3}}{3}+\frac{N^{2}}{2}+\frac{N}{6}} e^{-\frac{N^{3}}{9}+\frac{N}{12}}\right)^{4}, \\
\prod_{n=1}^{2 N} n \sim \sqrt{2 \pi}(2 N)^{2 N+\frac{1}{2}} e^{-2 N} \\
\prod_{n=1}^{N}(2 n)=2^{N} \prod_{n=1}^{N} n \sim \sqrt{2 \pi} N^{N+\frac{1}{2}} e^{-N} 2^{N},
\end{gathered}
$$

we have

$$
\begin{aligned}
A_{N} \sim & \exp \left(\frac{1}{24}+\left(\frac{N^{3}}{3}+\frac{N^{2}}{2}+\frac{N}{6}\right)+\frac{N}{12}\right) \\
& \times e^{\frac{9}{4} \zeta^{\prime}(-2)} N^{N^{3}-\frac{N}{4}} 2^{N^{3}-\frac{N}{4}} e^{-\frac{N^{3}}{3}-\frac{N}{8}} \\
& \times N^{\frac{N}{4}} 2^{\frac{N}{4}+\frac{1}{8}} e^{-\frac{N}{4}} \times(2 N+1)^{-N^{3}} .
\end{aligned}
$$

Hence, combining with

$$
\begin{aligned}
(2 N+1)^{-N^{3}} & =(2 N)^{-N^{3}}\left(1+\frac{1}{2 N}\right)^{-N^{3}} \\
& =(2 N)^{-N^{3}} \exp \left(-N^{3} \log \left(1+\frac{1}{2 N}\right)\right) \\
& \sim(2 N)^{-N^{3}} \exp \left(-N^{3}\left(\frac{1}{2 N}-\frac{1}{2}\left(\frac{1}{2 N}\right)^{2}+\frac{1}{3}\left(\frac{1}{2 N}\right)^{3}\right)\right) \\
& =(2 N)^{-N^{3}} \exp \left(-\frac{N^{2}}{2}+\frac{N}{8}-\frac{1}{24}\right),
\end{aligned}
$$

we obtain the desired result

$$
\lim _{N \rightarrow \infty} A_{N}=2^{\frac{1}{8}} \exp \left(\frac{9}{4} \zeta^{\prime}(-2)\right)
$$


Remarks. (1) The fact that $\mathcal{S}_{2}\left(\frac{1}{2}\right)=\sqrt{2}$ is also proved as Theorem 3 and we get (11) again from (4). In fact:

$$
\begin{aligned}
\mathcal{S}_{2}\left(\frac{1}{2}\right) & =e^{\frac{1}{2}} \prod_{n=1}^{\infty}\left(\left(\frac{1-\frac{1}{2 n}}{1+\frac{1}{2 n}}\right)^{n} e\right) \\
& =\lim _{N \rightarrow \infty} e^{\frac{1}{2}} \prod_{n=1}^{N}\left(\left(\frac{2 n-1}{2 n+1}\right)^{n} e\right) \\
& =\lim _{N \rightarrow \infty}\left(e^{\frac{1}{2}}\left(\frac{1}{3}\right)^{1}\left(\frac{3}{5}\right)^{2}\left(\frac{5}{7}\right)^{3} \cdots\left(\frac{2 N-1}{2 N+1}\right)^{N} e^{N}\right) \\
& =\lim _{N \rightarrow \infty}\left(e^{\frac{1}{2}} \frac{3 \cdot 5 \cdots(2 N-1)}{(2 N+1)^{N}} e^{N}\right) \\
& =\lim _{N \rightarrow \infty}\left(e^{\frac{1}{2}} \frac{(2 N) !}{N ! 2^{2 N} N^{N}\left(1+\frac{1}{2 N}\right)^{N}} e^{N}\right) \\
& =\sqrt{2}
\end{aligned}
$$

by the (usual) Stirling formula.

(2) The case of $\mathcal{S}_{3}(1 / 2)$ is similar by using the generalized Stirling's formula:

$$
\begin{aligned}
\mathcal{S}_{3}\left(\frac{1}{2}\right) & =e^{\frac{1}{8}} \prod_{n=1}^{\infty}\left(\left(1-\frac{1}{4 n^{2}}\right)^{n^{2}} e^{\frac{1}{4}}\right) \\
& =\lim _{N \rightarrow \infty} e^{\frac{1}{8}} \prod_{n=1}^{N}\left(\left(1-\frac{1}{4 n^{2}}\right)^{n^{2}} e^{\frac{1}{4}}\right) \\
& =\lim _{N \rightarrow \infty} e^{\frac{N}{4}+\frac{1}{8}} \prod_{n=1}^{N}\left(\frac{(2 n-1)(2 n+1)}{(2 n)^{2}}\right)^{n^{2}} \\
& =\lim _{N \rightarrow \infty} e^{\frac{N}{4}+\frac{1}{8}} \frac{\left(\prod_{n=1}^{2 N} n^{n^{2}}\right)^{1 / 2}}{\left(\prod_{n=1}^{N} n^{n^{2}}\right)^{4}} \times\left(\frac{(2 N) !}{2^{N} N !}\right)^{\frac{1}{2}} \times \frac{(2 N+1)^{N^{2}}}{2^{4\left(1^{2}+\cdots+N^{2}\right)}} \\
& =2^{\frac{1}{4}} \exp \left(\frac{7}{2} \zeta^{\prime}(-2)\right) .
\end{aligned}
$$

Thus we obtain Euler's formula (2) via (5).

(3) Except for $\mathcal{S}_{2}(1 / 2)=\sqrt{2}$ we do not know the algebraicity of $\mathcal{S}_{r}(1 / 2)$ for $r \geq 2$. In fact we cannot deny even the optimistic expectation $\mathcal{S}_{r}(1 / 2) \in 2^{\mathrm{Q}}$.

\section{ACKNOWLEDGEMENT}

We thank the referees for their suggestions to refine the presentation.

\section{REFERENCES}

[E1] L. Euler "De summis serierum numeros Bernoullianos involventium" Novi commentarii academiae scientiarum Petropolitanae 14 (1769) 129-167 [Opera Omnia I-15, pp.91-130].

[E2] L. Euler "Exercitationes analyticae" Novi commentarii academiae scientiarum Petropolitanae 17 (1772) 173-204 [Opera Omnia I-15, pp.131-167]. 
[E3] L. Euler "Remarques sur un beau rapport entre les séries des puissances tant directes que réciproques" Mémoires de l'académie des sciences de Berlin 17 (1761) 83-106 (Lu en 1749) [Opera Omnia I-15, pp. 70-90].

[H] G.H. Hardy "Divergent Series" Oxford Univ. Press 1949. MR0030620 (11:25a)

[K1] N. Kurokawa "Multiple sine functions and Selberg zeta functions" Proc. Japan Acad. 67A (1991) 61-64. MR.1105522 (92d:11094)

[K2] N. Kurokawa "Multiple zeta functions: an example" Adv. Stud. in Pure Math. 21 (1992) 219-226. MR 1210791 (94f:11084)

[KK1] N. Kurokawa and S. Koyama "Multiple sine functions" Forum Math. 15 (2003) 839-876. MR2010282 (2004g:11077)

[KK2] S. Koyama and N. Kurokawa "Kummer's formula for multiple gamma functions" J. Ramanujan Math. Soc. 18 (2003) 87-107. MR.1966530 (2004a:33003)

[KOW] N. Kurokawa, H. Ochiai and M. Wakayama "Multiple trigonometry and zeta functions" J. Ramanujan Math. Soc. 17 (2002) 101-113. MF.1913896 (2003f:11132)

[KW] N. Kurokawa and M. Wakayama "On $\zeta(3)$ " J. Ramanujan Math. Soc. 16 (2001) 205-214. MR1863604 (2002i:11083)

$[\mathrm{M}] \quad$ Yu. I. Manin "Lectures on zeta functions and motives (according to Deninger and Kurokawa)" Astérisque 228 (1995) 121-163. MR1330931 (96d:11076)

2-5-27 Hayabuchi, Tsuzuki-Ku, Yokohama 224-0025, Japan

E-mail address: koyama@tmtv.ne.jp

Department of Mathematics, Tokyo Institute of Technology, 152-8551, Tokyo, Japan

E-mail address: kurokawa@math.titech.ac.jp 